\title{
Long-term pain outcomes in elderly patients with trigeminal neuralgia: comparison of first-time microvascular decompression and stereotactic radiosurgery
}

\author{
Kunal P. Raygor, MD, ${ }^{1}$ Anthony T. Lee, MD, PhD, ${ }^{1}$ Noah Nichols, BA, ${ }^{1}$ Doris D. Wang, MD, PhD, ${ }^{1}$ \\ Mariann M. Ward, NP, MS, ${ }^{1}$ Nicholas M. Barbaro, MD, ${ }^{2}$ and Edward F. Chang, MD1 \\ 1Department of Neurological Surgery, University of California, San Francisco, California; and 2Department of Neurological \\ Surgery, University of Texas at Austin, Texas
}

OBJECTIVE Common surgical treatments for trigeminal neuralgia (TN) include microvascular decompression (MVD) and stereotactic radiosurgery (SRS). The use of MVD in elderly patients has been described but has yet to be prospectively compared to SRS, which is well-tolerated and noninvasive. The authors aimed to directly compare long-term pain control and adverse event rates for first-time surgical treatments for idiopathic TN in the elderly.

METHODS A prospectively collected database was reviewed for TN patients who had undergone treatment between 1997 and 2017 at a single institution. Standardized collection of preoperative demographics, surgical procedure, and postoperative outcomes was performed. Data analysis was limited to patients over the age of 65 years who had undergone a first-time procedure for the treatment of idiopathic TN with at least 1 year of follow-up.

RESULTS One hundred ninety-three patients meeting the study inclusion criteria underwent surgical procedures for TN during the study period (54 MVD, 24 MVD+Rhiz, 115 SRS). In patients in whom an artery was not compressing the trigeminal nerve during MVD, a partial sensory rhizotomy (MVD+Rhiz) was performed. Patients in the SRS cohort were older than those in the MVD and MVD+Rhiz cohorts (mean \pm SD, $79.2 \pm 7.8$ vs $72.9 \pm 5.7$ and $70.9 \pm 4.8$ years, respectively; $p<0.0001)$ and had a higher mean Charlson Comorbidity Index (3.8 \pm 1.1 vs $3.0 \pm 0.9$ and $2.9 \pm 1.0$, respectively; $p<0.0001$ ). Immediate or short-term postoperative pain-free rates (Barrow Neurological Institute [BNI] pain intensity score I) were $98.1 \%$ for MVD, $95.8 \%$ for MVD+Rhiz, and $78.3 \%$ for SRS ( $p=0.0008)$. At the last follow-up, $72.2 \%$ of MVD patients had a favorable outcome (BNI score I-IIla) compared to $54.2 \%$ and $49.6 \%$ of MVD+Rhiz and SRS patients, respectively ( $p=0.02)$. In total, $0(0 \%)$ SRS, $5(9.3 \%)$ MVD, and $1(4.2 \%)$ MVD+Rhiz patients developed any adverse event. Multivariate Cox proportional hazards analysis demonstrated that procedure type $(p=0.001)$ and postprocedure sensory change $(p=0.003)$ were statistically significantly associated with pain control.

CONCLUSIONS In this study cohort, patients who had undergone MVD had a statistically significantly longer duration of pain freedom than those who had undergone MVD+Rhiz or SRS as their first procedure. Fewer adverse events were seen after SRS, though the MVD-associated complication rate was comparable to published rates in younger patients. Overall, the results suggest that both MVD and SRS are effective options for the elderly, despite their advanced age. Treatment choice can be tailored to a patient's unique condition and wishes.

https://thejns.org/doi/abs/10.3171/2020.7.FOCUS20446

KEYWORDS trigeminal neuralgia; microvascular decompression; rhizotomy; stereotactic radiosurgery; elderly; surgical outcome; long-term outcome

$\mathrm{T}$ RIGEMINAL neuralgia (TN) is characterized by paroxysmal, lancinating facial pain in the trigeminal nerve's sensory distribution. Common surgical treatments for TN include ablative procedures such as stereotactic radiosurgery (SRS) and nonablative surgical microvascular decompression (MVD). Ablative therapies utilize nerve desensitization and injury to resolve pain, whereas nonablative procedures aim to relieve pain by removing the causative physical compression of the trigeminal nerve by offending vasculature. In cases in which no arterial compression of

ABBREVIATIONS BNI = Barrow Neurological Institute; $\mathrm{CCI}=$ Charlson Comorbidity Index; FS = Frailty Score; MVD = microvascular decompression; MVD+Rhiz = MVD with partial sensory rhizotomy; SRS = stereotactic radiosurgery; TN = trigeminal neuralgia; UCSF = University of California, San Francisco.

SUBMITTED May 27, 2020. ACCEPTED July 17, 2020.

INCLUDE WHEN CITING DOI: 10.3171/2020.7.FOCUS20446. 
the trigeminal nerve is found on operative exposure, partial sensory rhizotomy (MVD+Rhiz) is a reliable alternative for producing pain relief. ${ }^{1}$

Management of TN in the elderly is particularly challenging ${ }^{2}$ but is very important given its increased tendency to occur in this population. There is growing literature describing the efficacy of both MVD and SRS in the elderly, but prospective comparisons with extended follow-up are lacking. The efficacy of MVD for TN is well established, ${ }^{3}$ and several series have described its effectiveness in elderly patients, with pain-free rates and adverse event profiles similar to those in younger patients. ${ }^{4-7}$ Despite this, the use of MVD in the elderly is often questioned given concerns about complications, particularly in the perioperative period. In addition, SRS has been successfully used in elderly patients, ${ }^{8}$ though the effect of age on SRSassociated outcomes remains controversial.9,10 Two prior retrospective studies comparing the use of MVD and SRS in elderly patients demonstrated a prolonged duration of pain freedom in the MVD cohort, but the studies were limited by their retrospective natures and short follow-up durations. ${ }^{11,12}$ For this reason, a direct prospective comparison of surgical outcomes for elderly patients would be valuable in guiding management.

Here, we report our institution's longitudinal experience in the surgical treatment of idiopathic TN in the elderly as contained in a prospectively collected database. Using standardized data collection and long-term followup, we performed a direct comparison of pain-control rates in patients over the age of 65 years with idiopathic TN who had undergone MVD, MVD+Rhiz, or SRS for first-time surgical treatment. By defining long-term pain control and complication rates for these procedures and identifying predictors of favorable outcomes, we hope to provide valuable information in guiding treatment selection in this patient population.

\section{Methods \\ Patient Selection}

Clinical data for consecutive patients undergoing surgical treatment for TN at the University of California, San Francisco (UCSF) have been prospectively collected since 1997. This study is an analysis of all patients over the age of 65 who underwent surgical treatment (MVD, MVD+Rhiz, or SRS) performed by two surgeons (N.M.B. and E.F.C.) between 1997 and 2017. Inclusion criteria were as follows: patients with idiopathic TN without mass lesions or multiple sclerosis, those with classic type 1 trigeminal pain, ${ }^{13-15}$ those undergoing first-time surgical treatment for TN, age $>65$ years at the time of surgery, $>1$ year of follow-up, and sufficient preoperative and follow-up data. The decision to perform MVD or SRS was based on the patient's request after a discussion of perceived patient-specific risks and benefits, taking into account each patient's medical comorbidities and risks related to anesthesia and procedural intervention, including a history of any bleeding diathesis; use of anticoagulation/antiplatelet medications; severe cardiac, pulmonary, or renal comorbidities; and medical frailty. All research protocols were approved by the UCSF Institutional Review Board (Human Research Protection Program).

\section{Data Collection}

Clinical information was prospectively collected starting at the time that patients underwent evaluation for TN surgery at UCSF. Prospectively recorded variables included patient demographics, symptom duration, symptom location, symptom features, TN medications, relevant family and medical history, baseline physical examination findings, and imaging findings. The Charlson Comorbidity Index (CCI) and Frailty Score $(\mathrm{FS})^{16}$ were calculated based on the patient's medical history. The degree of preoperative frailty was dichotomized as low or high based on a cutoff FS of 4, as described previously. ${ }^{16}$ Follow-up visits were usually scheduled at 1, 3, 6, and 12 months postoperatively, with additional visits scheduled as needed. Variables collected on follow-up visits included previous treatments received, medications, TN pain description, sensory disturbances, as well as examination findings. All patients presented at least for the 1-month postprocedure visit; however, if patients did not undergo additional regular follow-up, telephone interviews were performed and the same variables (excluding physical examination findings) were recorded.

\section{Outcome Measures}

The primary outcome was dichotomized as favorable or unfavorable based on the Barrow Neurological Institute (BNI) pain intensity score ${ }^{17}$ at the last follow-up, as described previously. ${ }^{18}$ Briefly, BNI scores of I-IIIa constituted favorable outcomes, whereas BNI scores of IIIb-V were deemed to be unfavorable. Patients were considered to be pain free if they had no TN pain and were off medications (BNI score I). Secondary outcomes included adverse events and postprocedure sensory changes.

\section{Procedural Technique}

\section{Microvascular Decompression}

MVD was performed using previously described techniques. ${ }^{19-21}$ Briefly, after making a small retrosigmoid craniectomy, the trigeminal nerve was examined for vascular compression at the root entry zone or along its cisternal portion. Compressive arteries or veins were dissected away from the nerve, padded with Teflon felt, and secured with biological adhesive. In patients in whom no significant intraoperative vascular compression was visualized, a partial sensory rhizotomy was performed. Intraoperative brainstem auditory evoked potential monitoring was used in all patients.

\section{Stereotactic Radiosurgery}

SRS was performed using a Gamma Knife apparatus. The Leksell stereotactic head frame (Elekta Instruments AB) was applied under local anesthesia, and patients underwent stereotactic MRI. High-resolution T2-weighted images or T2-type fast imaging employing steady-state acquisition (FIESTA) sequences and contrast-enhanced T1-weighted images were used for target planning. The cisternal portion of the trigeminal nerve was targeted with a single isocenter using a 4-mm collimator. Maximum doses for SRS ranged from 70 to $80 \mathrm{~Gy}$. The majority of patients $(95[82.6 \%])$ received $80 \mathrm{~Gy}$, while $12(10.4 \%)$ received 75 Gy, and 8 (7\%) received $70 \mathrm{~Gy}$. 


\section{Statistical Analysis}

All statistical analyses were performed using JMP Pro version 14 (SAS Institute). Continuous predictor and outcome variables were compared among the three cohorts using either the parametric ANOVA or the nonparametric Kruskal-Wallis test, as appropriate. Categorical variables were compared with Pearson's chi-square test or Fisher's exact test, as appropriate. The duration of time from surgery to an unfavorable outcome for the various surgical procedures was plotted using Kaplan-Meier survival analyses, and statistical significance was measured using the log-rank test. Multivariate Cox proportional hazards modeling was used to determine the effect of multiple variables on the time to pain recurrence. Only variables with a $\mathrm{p}$ value $<0.2$ on univariate analysis were used in the multivariate regression model to avoid overfitting. Univariate analyses were performed to find predictors of outcome for each procedure type. The threshold for significance was set at a $p$ value of 0.05 .

\section{Results}

Between 1997 and 2017, a total of 193 elderly patients underwent first-time procedural intervention for $\mathrm{TN}$ at UCSF and met all study inclusion criteria, with 54 undergoing MVD, 24 undergoing MVD+Rhiz, and 115 undergoing SRS. Patient demographics (Table 1) demonstrated that patients in the SRS cohort were older than those in the MVD and MVD+Rhiz cohorts (mean \pm SD, $79.2 \pm$ 7.8 vs $72.9 \pm 5.7$ and $70.9 \pm 4.8$ years, respectively; $\mathrm{p}<$ $0.0001)$ and had a greater mean CCI $(3.8 \pm 1.1$ vs $3.0 \pm$ 0.9 and $2.9 \pm 1.0$, respectively; $\mathrm{p}<0.0001$ ). No MVD or MVD+Rhiz patients had a high degree of preoperative frailty, while $10(8.7 \%)$ SRS patients did $(\mathrm{p}=0.03)$. SRS patients also had longer preprocedure symptom duration (119.6 \pm 98 months vs $72.9 \pm 68.7$ months and $62.3 \pm 43.4$ months, respectively; $p=0.0005$ ). There were no differences in sex, TN laterality, TN distribution, family history of TN, preprocedure sensory disturbance, or followup duration.

\section{Procedural Outcomes}

Pain relief outcomes were analyzed for each cohort of patients (Table 2). After their procedure, 53 (98.1\%) MVD patients, 23 (95.8\%) MVD+Rhiz patients, and 90 (78.3\%) SRS patients became pain free (BNI score I; $p=0.0008$ ). The median (IQR) time to pain freedom was $0(0,0)$ weeks for the MVD and MVD+Rhiz cohorts compared to $25.9(6.9,52.9)$ weeks for the SRS cohort $(\mathrm{p}<0.0001)$. At the last follow-up, MVD patients (72.2\%) were more likely to have a favorable outcome (BNI score I-IIIa) than the MVD+Rhiz $(54.2 \%)$ or SRS $(49.6 \%)$ patients $(\mathrm{p}=0.02)$. Kaplan-Meier survival analysis depicts the time from the procedure to the development of an unfavorable outcome (Fig. 1). The median (IQR) duration of a favorable outcome was not reached in the MVD cohort but was 44.7 $(26.8,64.6)$ months in the MVD+Rhiz cohort and 66.2 $(7.8,139.5)$ months in the SRS cohort $(\mathrm{p}=0.03)$. Patients who underwent MVD were significantly less likely to develop postprocedure sensory changes than those undergoing MVD+Rhiz or SRS ( $\mathrm{p}<0.0001)$.
TABLE 1. Demographic data and clinical characteristics of patients in the MVD, MVD+Rhiz, and SRS cohorts

\begin{tabular}{|c|c|c|c|c|}
\hline Variable & $\begin{array}{c}\text { MVD } \\
(n=54)\end{array}$ & $\begin{array}{c}\text { MVD+Rhiz } \\
(n=24)\end{array}$ & $\begin{array}{c}\text { SRS } \\
(n=115)\end{array}$ & $p$ Value \\
\hline Male sex & $21(38.9)$ & $4(16.7)$ & $42(36.5)$ & 0.13 \\
\hline TN laterality & & & & 0.74 \\
\hline Rt & $32(59.3)$ & $12(50.0)$ & $66(57.4)$ & \\
\hline Lt & $22(40.7)$ & $12(50.0)$ & $49(42.6)$ & \\
\hline TN distribution & & & & 0.79 \\
\hline V1 & $5(9.3)$ & $2(8.3)$ & $8(7.0)$ & \\
\hline V2 & $10(18.5)$ & $5(20.8)$ & 20 (17.4) & \\
\hline V3 & $14(25.9)$ & 7 (29.2) & $26(22.6)$ & \\
\hline V1 \& V2 & $10(18.5)$ & $3(12.5)$ & $15(13.0)$ & \\
\hline V2 \& V3 & $10(18.5)$ & $7(29.2)$ & $31(27.0)$ & \\
\hline V1-V3 & $5(9.3)$ & $0(0)$ & $15(13.0)$ & \\
\hline $\begin{array}{l}\text { Family history } \\
\text { of TN }\end{array}$ & $0(0)$ & $0(0)$ & $7(6.1)$ & 0.18 \\
\hline Mean age in yrs & $72.2 \pm 5.7$ & $70.9 \pm 4.8$ & $79.2 \pm 7.8$ & $<0.0001$ \\
\hline $\begin{array}{l}\text { Mean preop } \\
\text { symptom } \\
\text { duration in mos }\end{array}$ & $72.9 \pm 68.7$ & $62.3 \pm 43.4$ & $119.6 \pm 98.0$ & 0.0005 \\
\hline $\begin{array}{l}\text { Preop sensory } \\
\text { disturbance }\end{array}$ & $0(0)$ & $1(4.2)$ & $10(8.7)$ & 0.07 \\
\hline Mean CCl & $3.0 \pm 0.9$ & $2.9 \pm 1.0$ & $3.8 \pm 1.1$ & $<0.0001$ \\
\hline Preop frailty & & & & 0.03 \\
\hline Low $($ FS <4) & $54(100)$ & $24(100)$ & $105(91.3)$ & \\
\hline High (FS $\geq 4$ ) & $0(0)$ & $0(0)$ & $10(8.7)$ & \\
\hline Mean FU in mos & $55.6 \pm 32.7$ & $49.9 \pm 39.9$ & $57.4 \pm 43.7$ & 0.71 \\
\hline
\end{tabular}

$\mathrm{FU}=$ follow-up; $\mathrm{n}=$ number of patients.

Values are expressed as number (\%) or as mean $\pm \mathrm{SD}$, unless indicated otherwise. Boldface type indicates statistical significance.

Procedural complications were also analyzed for each cohort (Table 2). In total, 5 (9.3\%) MVD patients, 1 (4.2\%) MVD+Rhiz patient, and $0(0 \%)$ SRS patients developed any adverse event $(p=0.005)$. There were no cases of painful dysesthesia or anesthesia dolorosa in the SRS cohort. Of the 6 adverse events occurring in the MVD and MVD+Rhiz cohorts, 4 were CSF leakages ( 3 in the MVD cohort, 1 in the MVD+Rhiz cohort) requiring lumbar CSF diversion and/ or surgical exploration and dural closure. The remaining 2 complications in the MVD cohort were 1 wound infection requiring debridement and 1 posterior fossa subdural hematoma requiring evacuation. There were no observed perioperative cardiopulmonary or thromboembolic complications and no procedure-related deaths. After combining all patients with any adverse event from both the MVD and MVD+Rhiz cohorts, univariate analysis was performed to determine any variables associated with the development of an adverse event. While the mean CCI was higher in the patients who developed adverse events ( $3.5 \pm 1.8$ vs $2.9 \pm 0.8$, $\mathrm{p}=0.14$ ), the difference did not reach statistical significance. All other demographic and preoperative variables, including sex, TN laterality, TN distribution, family history of TN, age, preprocedure symptom duration, and preprocedure sensory disturbance, had no statistically significant association with adverse events. 
TABLE 2. Pain relief outcomes and adverse events among patients in the MVD, MVD+Rhiz, and SRS cohorts

\begin{tabular}{lcccc}
\hline \multicolumn{1}{c}{ Variable } & MVD $(\mathrm{n}=54)$ & MVD+Rhiz $(\mathrm{n}=24)$ & SRS $(\mathrm{n}=115)$ & $\mathrm{p}$ Value \\
\hline Pain-free status, BNI I* & $53(98.1)$ & $23(95.8)$ & $90(78.3)$ & $\mathbf{0 . 0 0 0 8}$ \\
\hline Median time to BNI I in wks (IQR) & $0(0,0)$ & $0(0,0)$ & $25.9(6.9,52.9)$ & $<0.0001$ \\
\hline BNI pain intensity score at last FU & & & & 0.003 \\
I & $37(68.5)$ & $11(45.8)$ & $53(46.1)$ & $4(3.5)$ \\
II & $2(3.7)$ & $0(0)$ & $0(0)$ \\
IIIa & $0(0)$ & $2(8.3)$ & $5(4.3)$ \\
IIIb & $1(1.9)$ & $2(8.3)$ & $5(4.3)$ \\
IV & $3(5.6)$ & $0(0)$ & $48(41.7)$ & \\
V & $11(20.4)$ & $9(37.5)$ & $57(49.6)$ & 0.02 \\
\hline Favorable outcome at last FU, BNI I-IIla & $39(72.2)$ & $13(54.2)$ & $66.2(7.8,139.5)$ & 0.03 \\
\hline Median duration of favorable outcome in mos (IQR) & $-(35.4,-)$ & $44.7(26.8,64.6)$ & $54(47.0)$ & $<0.0001$ \\
\hline Postop sensory change & $8(14.8)$ & $15(62.5)$ & $0(0)$ & 0.005 \\
Any adverse event & $5(9.3)$ & $1(4.2)$ & $0(0)$ & \\
CSF leakage & $3(5.6)$ & $1(4.2)$ & $0(0)$ & \\
Subdural hematoma & $1(1.9)$ & $0(0)$ & $0(0)$ & \\
Wound infection & $1(1.9)$ & $0(0)$ & $0(0)$ & \\
Anesthesia dolorosa & $0(0)$ & $0(0)$ & \\
\hline
\end{tabular}

Values are expressed as number (\%), unless indicated otherwise. Boldface type indicates statistical significance.

${ }^{*}$ Anytime in the first 4 weeks after surgery.

Overall mortality in our elderly cohort was also assessed. At the time of the last follow-up, 1 (1.3\%) patient in the combined MVD and MVD+Rhiz cohorts had died. In the SRS cohort, $11(9.6 \%)$ patients had died by the last follow-up. All mortalities were unrelated to the procedure performed and occurred at least 1 year from the procedure date.

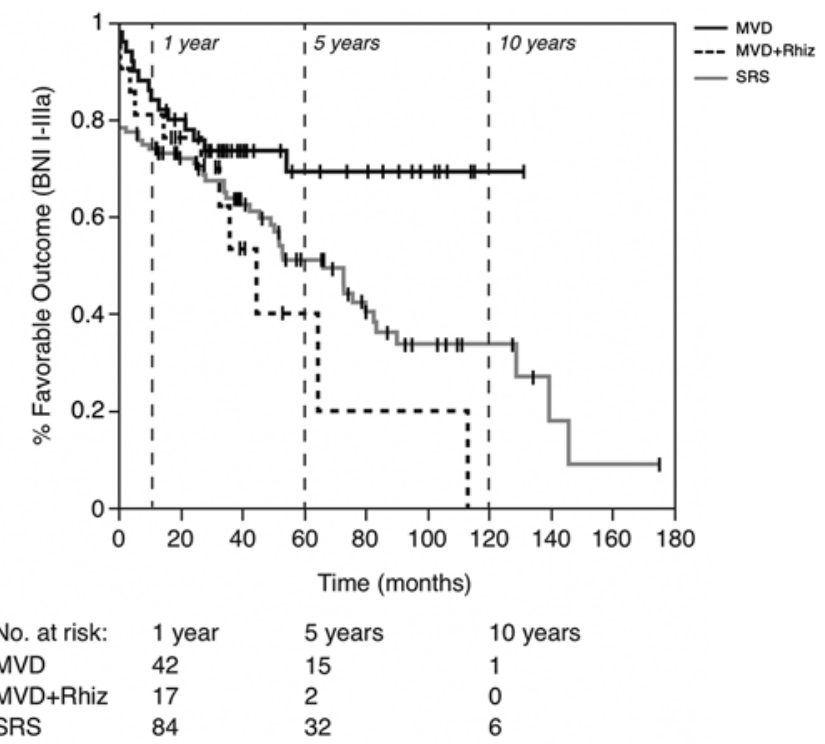

FIG. 1. Pain recurrence by procedure type: MVD versus MVD+Rhiz versus SRS. Kaplan-Meier survival curves for each cohort are shown, with tick marks representing censored events. Values in the graph represent the number of patients from each group reaching time points indicated by the dashed vertical lines (1,5, and 10 years).

\section{Predictors of a Favorable Outcome}

Overall predictors of favorable and unfavorable outcomes were analyzed (Table 3). On univariate analysis, only the procedure type was statistically significantly associated with a favorable outcome $(p=0.02)$. The presence of a postprocedure sensory change tended to be associated with a favorable outcome, but the association was not statistically significant $(p=0.06)$. Sex, TN laterality, TN distribution, age, preprocedure symptom duration, family history of TN, presence of preprocedural sensory disturbance, comorbidities, and degree of preoperative frailty were not associated with a favorable outcome at the last follow-up. To determine what variables best predicted the time to an unfavorable outcome, a multivariate Cox proportional hazards analysis was performed (Table 4). The use of MVD, as compared to MVD+Rhiz (HR 3.59, 95\% CI 1.22-10.56) or SRS (HR 3.73, 95\% CI 1.76-7.90), was associated with a prolonged duration of a favorable outcome ( $\mathrm{p}=0.001)$. The development of a postprocedure sensory change (HR 2.66, 95\% CI 1.38-5.13, p = 0.003) was also associated with a prolonged duration of a favorable outcome.

To further determine specific predictors of a favorable outcome by procedure type, univariate analyses were performed for the MVD cohort (Table 5), MVD+Rhiz cohort (Table 6), and SRS cohort (Table 7). Briefly, there were no statistically significant associations between individual predictors and a favorable outcome in the MVD and MVD+Rhiz cohorts. Specifically, in the MVD cohort, female patients were more likely to have favorable outcomes than males ( $69.2 \%$ vs $57.1 \%$, respectively), though the difference between the two sexes was not statistically significant $(\mathrm{p}=0.07)$. Although only $32(59.3 \%)$ MVD patients had MRI evidence of compression of the trigeminal nerve, 
TABLE 3. Univariate analysis of favorable outcome at the last follow-up

\begin{tabular}{|c|c|c|c|c|}
\hline Variable & Total & Favorable Outcome, BNI I-IIla & Unfavorable Outcome, BNI IIIb-V & $\mathrm{p}$ Value \\
\hline No. of patients & 193 & 109 & 84 & - \\
\hline Procedure type & & & & 0.02 \\
\hline MVD & $54(28.0)$ & $39(72.2)$ & $15(27.8)$ & \\
\hline MVD+Rhiz & $24(12.4)$ & $13(54.2)$ & $11(45.8)$ & \\
\hline SRS & $115(59.6)$ & $57(49.6)$ & $58(50.4)$ & \\
\hline Male sex & $67(34.7)$ & $35(52.2)$ & $32(47.8)$ & 0.45 \\
\hline TN laterality & & & & 0.97 \\
\hline Rt & $110(57.0)$ & $62(56.4)$ & $48(43.6)$ & \\
\hline $\mathrm{Lt}$ & $83(43.0)$ & $47(56.6)$ & $36(43.4)$ & \\
\hline TN distribution & & & & 0.62 \\
\hline V1 & $15(7.8)$ & $8(53.3)$ & $7(46.7)$ & \\
\hline V2 & $35(18.1)$ & $17(48.6)$ & $18(51.4)$ & \\
\hline V3 & $47(24.4)$ & $24(51.1)$ & $23(48.9)$ & \\
\hline V1 \& V2 & $28(14.5)$ & $17(60.7)$ & $11(39.3)$ & \\
\hline $\mathrm{V} 2 \& \mathrm{~V} 3$ & $48(24.9)$ & $29(60.4)$ & $19(39.6)$ & \\
\hline V1-V3 & $20(10.4)$ & $14(70.0)$ & $6(30.0)$ & \\
\hline Mean age in yrs & $76.2 \pm 7.8$ & $76.3 \pm 7.6$ & $76.1 \pm 8.1$ & 0.82 \\
\hline Mean preop symptom duration in mos & $98.8 \pm 88.2$ & $95.9 \pm 83.4$ & $102.3 \pm 90.8$ & 0.62 \\
\hline Family history of TN & $8(4.1)$ & $3(37.5)$ & $5(62.5)$ & 0.30 \\
\hline Preop sensory disturbance & $11(5.7)$ & $5(45.5)$ & $6(54.5)$ & 0.54 \\
\hline Mean CCl & $3.5 \pm 1.1$ & $3.5 \pm 1.1$ & $3.5 \pm 1.1$ & 0.97 \\
\hline Preop frailty & & & & 0.34 \\
\hline Low frailty $(\mathrm{FS}<4)$ & $183(94.8)$ & $105(57.4)$ & $78(42.6)$ & \\
\hline High frailty (FS $\geq 4$ ) & $10(5.2)$ & $4(40.0)$ & $6(60.0)$ & \\
\hline Postop sensory change & $77(39.9)$ & $50(64.9)$ & $27(35.1)$ & 0.06 \\
\hline
\end{tabular}

Values are expressed as number (\%) or as mean \pm SD, unless indicated otherwise. Boldface type indicates statistical significance.

all 54 patients had intraoperative evidence of compression. Among the MVD patients, $72.2 \%$ had single artery compression of the nerve, $7.4 \%$ had single vein compression of the nerve, and $20.4 \%$ had combined arterial and venous compression of the nerve, although the compressing vessel was not associated with a favorable outcome $(\mathrm{p}=0.77)$. In the MVD+Rhiz cohort, $12(50.0 \%)$ patients had MRI evidence of nerve compression, but 21 (87.5\%) had evidence of mild intraoperative compression. Despite this mild compression, the operating surgeon (N.M.B. or E.F.C.) determined intraoperatively that the compression was not severe, so a partial sensory rhizotomy was performed. Evidence of mild intraoperative compression tended to be associated with a favorable outcome, though

TABLE 4. Multivariate Cox proportional hazards analysis of duration of favorable outcome

\begin{tabular}{llc}
\hline \multicolumn{1}{c}{ Predictor Variable } & HR $(95 \% \mathrm{Cl})$ & $\mathrm{p} \mathrm{Value}$ \\
\hline Procedure & & $\mathbf{0 . 0 0 1}$ \\
MVD vs MVD+Rhiz & $3.59(1.22-10.56)$ & \\
MVD vs SRS & $3.73(1.76-7.90)$ & \\
MVD+Rhiz vs SRS & $1.04(0.42-2.58)$ & \\
\hline Postprocedure sensory change & $2.66(1.38-5.13)$ & $\mathbf{0 . 0 0 3}$ \\
\hline
\end{tabular}

Boldface type indicates statistical significance. the association was not statistically significant $(\mathrm{p}=0.08)$. The nature of the compressing vessel in this cohort was also not associated with a favorable outcome $(\mathrm{p}=0.11)$.

In the SRS cohort, the radiation dose ranged from 70 to $80 \mathrm{~Gy}$ and was associated with a favorable outcome, with $55.8 \%$ of patients who received 80 Gy having a favorable outcome compared to only $25 \%$ and $12.5 \%$ of those who received 75 and $70 \mathrm{~Gy}$, respectively $(\mathrm{p}=0.01)$. In addition, the presence of a postprocedural sensory change was associated with a favorable outcome, with $64.8 \%$ of patients with postprocedure paresthesias having a favorable outcome $(\mathrm{p}=0.002)$.

\section{Discussion}

A direct comparison of ablative and nonablative procedures for the surgical treatment of medically refractory $\mathrm{TN}$ in the elderly has yet to be performed in a prospective manner. Here, we present our long-term experience with MVD, MVD+Rhiz, and SRS for the treatment of TN in the elderly. We found that MVD, as compared to MVD+Rhiz and SRS, was associated with a longer duration of a favorable outcome and that the presence of postprocedure sensory changes was predictive of better outcomes in the SRS cohort. Importantly, we also found that patients who had undergone SRS had a greater mean CCI and a higher degree of preoperative frailty compared to those of patients who had undergone MVD or MVD+Rhiz. While such in- 
TABLE 5. Univariate analysis of MVD outcomes

\begin{tabular}{|c|c|c|c|c|}
\hline Variable & Total & Favorable Outcome, BNI I-IIla & Unfavorable Outcome, BNI IIIb-V & $p$ Value \\
\hline No. of patients & 54 & 39 & 15 & - \\
\hline Sex & & & & 0.07 \\
\hline M & $21(38.9)$ & $12(57.1)$ & $9(42.9)$ & \\
\hline $\mathrm{F}$ & $33(61.1)$ & $27(81.8)$ & $6(18.2)$ & \\
\hline TN laterality & & & & 0.76 \\
\hline $\mathrm{Rt}$ & $32(59.3)$ & $24(75.0)$ & $8(25.0)$ & \\
\hline Lt & $22(40.7)$ & $15(68.2)$ & $7(31.8)$ & \\
\hline TN distribution & & & & 0.64 \\
\hline V1 & $5(9.3)$ & $5(100.0)$ & $0(0)$ & \\
\hline V2 & $10(18.5)$ & $7(70.0)$ & $3(30.0)$ & \\
\hline V3 & $14(25.9)$ & $8(57.1)$ & $6(42.9)$ & \\
\hline V1 \& V2 & $10(18.5)$ & $8(80.0)$ & $2(20.0)$ & \\
\hline V2 \& V3 & $10(18.5)$ & $7(70.0)$ & $3(30.0)$ & \\
\hline V1-V3 & $5(9.3)$ & $4(80.0)$ & $1(20.0)$ & \\
\hline Mean age in yrs & $72.2 \pm 5.7$ & $72.2 \pm 5.6$ & $72.4 \pm 6.1$ & 0.90 \\
\hline Mean preop symptom duration in mos & $72.9 \pm 68.7$ & $71.2 \pm 60.8$ & $77.9 \pm 89.7$ & 0.75 \\
\hline Median time to pain-free status in wks (IQR) & $0(0,0)$ & $0(0,0)$ & $0(0,2.3)$ & 0.45 \\
\hline MRI compression & $32(59.3)$ & $22(68.8)$ & $10(31.3)$ & 0.65 \\
\hline Intraop compression & $54(100)$ & $39(72.2)$ & $15(27.8)$ & - \\
\hline Compressing vessel & & & & 0.77 \\
\hline Artery only & $39(72.2)$ & $29(74.4)$ & $10(25.6)$ & \\
\hline Vein only & $4(7.4)$ & $3(75.0)$ & $1(25.0)$ & \\
\hline Artery + vein & $11(20.4)$ & $7(63.6)$ & $4(36.4)$ & \\
\hline None & $0(0)$ & $0(0)$ & $0(0)$ & \\
\hline Postop sensory change & $8(14.8)$ & $5(62.5)$ & $3(37.5)$ & 0.67 \\
\hline
\end{tabular}

Values are expressed as number (\%) or as mean $\pm \mathrm{SD}$, unless indicated otherwise.

dices were not specifically used to determine whether or not to pursue microsurgical intervention, they do show that preoperative evaluation can be used to separate patients based on measures of medical comorbidities and frailty, leading to excellent pain outcomes with reasonable adverse event profiles. Recent work has suggested that higher preoperative frailty can predict morbidity and mortality in patients undergoing cranial $^{16}$ or oncological ${ }^{22}$ neurosurgery. Thus, such indices should be further evaluated, as they may aid in counseling patients on treatment options.

\section{Pain Outcomes}

In our series, multivariate modeling showed that procedure type and the presence of postprocedure sensory changes in patients were associated with a longer duration of a favorable outcome. In particular, MVD, as compared to MVD+Rhiz or SRS, was associated with the longest duration of pain freedom, and there was no statistically significant difference between the MVD+Rhiz and SRS cohorts. Even so, both MVD and MVD+Rhiz were associated with high rates (98.2\% and $95.8 \%$, respectively) of short-term pain freedom (BNI score I). These rates are consistent with the existing literature for MVD in the elderly, which describes immediate or short-term BNI score I rates ranging from $70.3 \%$ to $97.2 \% .^{4-6,12,23-27}$ Direct comparisons with much of the literature is challenging, as outcome measures have not been standardized over the years; however, our finding of a favorable outcome in $72.2 \%$ of MVD patients at the last follow-up is similar to results in a recent retrospective comparison of MVD and SRS in the elderly by Oh et al., which demonstrated that $63.0 \%$ of patients had a BNI score of I or II at 2 years' follow-up. ${ }^{11}$ Other studies have described "completely pain-free" or "success" rates of $75 \%-87.5 \%$ after long-term follow-up., ${ }^{4,5,25}$ While MVD+Rhiz has not been studied in great detail in the elderly, long-term pain recurrence after rhizotomy across all patients has been reported to be similar to the long-term pain recurrence rates with SRS and other ablative procedures. ${ }^{10,28}$ Here, we noted that although patients undergoing MVD+Rhiz have high rates of pain freedom in the shortterm, only $54.2 \%$ of patients in that cohort had a favorable outcome at the last follow-up.

For patients in the SRS cohort, immediate pain-free rates approached $80 \%$, but favorable outcomes at the last follow-up dropped to $49.6 \%$. Similar rates of pain control have been described in recent radiosurgical series. ${ }^{8,29}$ Interestingly, Karam et al. found that an older age $(>70$ years old) was associated with favorable outcomes. ${ }^{29}$ While we did not find a statistically significant association between age and outcome in the SRS cohort, this finding may be related to our use of age as a continuous variable as opposed to a categorical one; in our cohort, the mean age of SRS patients with a favorable outcome was slightly higher than that of the SRS patients with unfavorable outcomes (80.2 vs 78.2 years). Even so, age itself may not be the primary factor driving pain relief, as a prior study 
TABLE 6. Univariate analysis of MVD+Rhiz outcomes

\begin{tabular}{|c|c|c|c|c|}
\hline Variable & Total & Favorable Outcome, BNI I-IIla & Unfavorable Outcome, BNI IIIb-V & p Value \\
\hline No. of patients & 24 & 13 & 11 & - \\
\hline Sex & & & & 0.85 \\
\hline M & $4(16.7)$ & $2(50.0)$ & $2(50.0)$ & \\
\hline $\mathrm{F}$ & $20(83.3)$ & $11(55.0)$ & $9(45.0)$ & \\
\hline TN laterality & & & & 0.41 \\
\hline Rt & $12(50.0)$ & $8(66.7)$ & $4(33.3)$ & \\
\hline $\mathrm{Lt}$ & $12(50.0)$ & $5(41.7)$ & $7(58.3)$ & \\
\hline TN distribution & & & & 0.96 \\
\hline V1 & $2(8.3)$ & $1(50.0)$ & $1(50.0)$ & \\
\hline V2 & $5(20.8)$ & $2(40.0)$ & $3(60.0)$ & \\
\hline V3 & $7(29.2)$ & $4(57.1)$ & $3(42.9)$ & \\
\hline V1 \& V2 & $3(12.5)$ & $2(66.7)$ & $1(33.3)$ & \\
\hline $\mathrm{V} 2 \& \mathrm{~V} 3$ & $7(29.2)$ & $4(57.1)$ & $3(42.9)$ & \\
\hline V1-V3 & $0(0)$ & $0(0)$ & $0(0)$ & \\
\hline Mean age in yrs & $70.9 \pm 4.8$ & $70.3 \pm 4.4$ & $71.7 \pm 5.3$ & 0.49 \\
\hline Mean preop symptom duration in mos & $62.3 \pm 43.4$ & $62.1 \pm 31.7$ & $62.5 \pm 55.9$ & 0.98 \\
\hline Family history of TN & $1(4.2)$ & $1(100)$ & $0(0)$ & 0.99 \\
\hline Preop sensory disturbance & $1(4.2)$ & $1(100)$ & $0(0)$ & 0.99 \\
\hline $\begin{array}{l}\text { Median time to pain-free status in } \\
\text { wks (IQR) }\end{array}$ & $0(0,0)$ & $0(0,0)$ & $0(0,0)$ & 0.26 \\
\hline MRI compression & $12(50.0)$ & $7(58.3)$ & $5(41.7)$ & 0.99 \\
\hline Intraop mild compression & $21(87.5)$ & $13(61.9)$ & $8(38.1)$ & 0.08 \\
\hline Compressing vessel & & & & 0.11 \\
\hline Artery only & $10(41.7)$ & $5(50.0)$ & $5(50.0)$ & \\
\hline Vein only & $9(37.5)$ & $7(77.8)$ & $2(22.2)$ & \\
\hline Artery + vein & $2(8.3)$ & $1(50.0)$ & $1(50.0)$ & \\
\hline None & $3(12.5)$ & $0(0)$ & $3(100.0)$ & \\
\hline Postop sensory change & $15(62.5)$ & $10(66.7)$ & $5(33.3)$ & 0.21 \\
\hline
\end{tabular}

Values are expressed as number (\%) or as mean $\pm \mathrm{SD}$, unless indicated otherwise.

by Mousavi et al. found that early radiosurgery, as compared to later radiosurgery, was associated with a longer duration of adequate pain control (defined as BNI scores I-IIIa). ${ }^{9}$ Though preprocedure symptom duration was not associated with a favorable outcome in our SRS cohort, this finding may be related to the fact that patients had an average of 10 years of symptoms prior to undergoing SRS, whereas the patients with the best outcome in the Mousavi et al. series underwent Gamma Knife radiosurgery within 3 years of diagnosis. We also found that radiation dose and postprocedure sensory change were associated with a favorable outcome in the SRS cohort, consistent with prior literature. . $^{8,10,18,30,31}$ We believe these findings should guide patients who are deemed to be poor candidates for open surgical decompression of the trigeminal nerve-in those patients who undergo SRS, favorable outcomes can still be expected if an 80-Gy maximal dose is delivered and postprocedure facial numbness is observed. Importantly, in elderly patients who do not obtain pain control after first-time SRS, a second SRS procedure may be beneficial. $^{8}$

Of course, in addition to SRS, other ablative procedures exist, such as percutaneous radiofrequency ablation of the gasserian ganglion (RFA), glycerol injection (PGZ), and balloon compression (PBC). Each of these procedures is effective at providing short-term pain relief, with $>98 \%$ of RFA and PBC patients obtaining complete pain relief by hospital discharge. ${ }^{32,33}$ Unfortunately, pain recurrence is common by 3 years,,$^{32}$ and rates of painful dysesthesias and anesthesia dolorosa remain high. ${ }^{34}$ Unlike SRS, these ablative procedures also require anesthesia, making them somewhat less desirable in the elderly.

\section{Adverse Events}

Despite having more durable pain control following MVD, patients undergoing open surgical intervention in this series had an increased rate of any adverse events compared to patients in the SRS cohort. Grouping the MVD and MVD+Rhiz patients together, we found a total adverse event rate of $7.7 \%$, including 4 CSF leakages, 1 wound infection, and 1 subdural hematoma requiring evacuation. These complications are consistent with the prior literature, though others have also described partial hearing loss, trigeminal hypesthesia, and meningitis after surgery. ${ }^{4,5} \mathrm{~A}$ recent meta-analysis also described increased mortality, stroke, and thromboembolism rates in elderly compared to nonelderly MVD patients, ${ }^{25}$ and a nationwide analysis of elderly TN patients undergoing MVD also revealed increased rates of cardiac and pulmonary 
TABLE 7. Univariate analysis of SRS outcomes

\begin{tabular}{|c|c|c|c|c|}
\hline Variable & Total & Favorable Outcome, BNI I-IIla & Unfavorable Outcome, BNI IIIb-V & p Value \\
\hline No. of patients & 115 & 57 & 58 & - \\
\hline Sex & & & & 0.94 \\
\hline M & $42(36.5)$ & $21(50.0)$ & $21(50.0)$ & \\
\hline $\mathrm{F}$ & $73(63.5)$ & $36(49.3)$ & $37(50.7)$ & \\
\hline TN laterality & & & & 0.31 \\
\hline Rt & $66(57.4)$ & $30(45.5)$ & $36(54.5)$ & \\
\hline $\mathrm{Lt}$ & $49(42.6)$ & $27(55.1)$ & $22(44.9)$ & \\
\hline TN distribution & & & & 0.38 \\
\hline V1 & $8(7.0)$ & $2(25.0)$ & $6(75.0)$ & \\
\hline $\mathrm{V} 2$ & $20(17.4)$ & $8(40.0)$ & $12(60.0)$ & \\
\hline V3 & $26(22.6)$ & $12(46.2)$ & $14(53.8)$ & \\
\hline V1 \& V2 & $15(13.0)$ & $7(46.7)$ & $8(53.3)$ & \\
\hline V2 \& V3 & $31(27.0)$ & $18(58.1)$ & $13(41.9)$ & \\
\hline V1-V3 & $15(13.0)$ & $10(66.7)$ & $5(33.3)$ & \\
\hline Mean age in yrs & $79.2 \pm 7.8$ & $80.2 \pm 7.2$ & $78.2 \pm 8.3$ & 0.16 \\
\hline Mean preop symptom duration in mos & $119.6 \pm 98.0$ & $123.1 \pm 101.0$ & $116.1 \pm 95.8$ & 0.71 \\
\hline Family history of TN & $7(6.1)$ & $2(28.6)$ & $5(71.4)$ & 0.44 \\
\hline Preop sensory disturbance & $105(91.3)$ & $53(50.5)$ & $52(49.5)$ & 0.74 \\
\hline SRS dose & & & & 0.01 \\
\hline 70 Gy & $8(7.0)$ & $1(12.5)$ & $7(87.5)$ & \\
\hline 75 Gy & $12(10.4)$ & $3(25.0)$ & $9(75.0)$ & \\
\hline $80 \mathrm{~Gy}$ & $95(82.6)$ & $53(55.8)$ & $42(44.2)$ & \\
\hline Median time to pain-free status in wks (IQR) & $25.9(6.9,52.9)$ & $26.9(7.9,53.4)$ & $24.9(6.1,52.9)$ & 0.86 \\
\hline Postop sensory change & $54(47.0)$ & $35(64.8)$ & $19(35.2)$ & 0.002 \\
\hline
\end{tabular}

Values are expressed as number (\%) or as mean \pm SD, unless indicated otherwise. Boldface type indicates statistical significance.

complications. ${ }^{26}$ In comparison, we observed no cases of perioperative cardiopulmonary or thromboembolic adverse events and no procedure-related deaths. The lack of life-threatening complications in our cohort may represent more stringent preoperative evaluation and optimization metrics before proceeding with MVD or MVD+Rhiz. Even so, patients with a high CCI tended to develop an adverse event. In contrast, no adverse events (including dysesthesias or anesthesia dolorosa) occurred in our SRS cohort. We believe that this increased rate of complications after MVD in the elderly should be discussed with patients, so that fully informed decisions can be made. In addition, we hope that future studies can more precisely model the preoperative risk for the development of adverse events, as such models would be able to more definitively guide treatment decisions in this patient population.

\section{Study Limitations}

Patients in our study were not randomized to treatment; therefore, some treatment bias is inherent to this study. For patients who were deemed to be poor operative candidates, SRS was recommended. This use of radiosurgery in more debilitated patients may result in lower morbidity and/or mortality in the MVD cohort, although the complication rate associated with MVD in this series is similar to previously published rates. While this does limit the generalizability of our results, we believe that our findings still provide relevant information regarding comparative outcomes for MVD and SRS in the elderly and can still help to guide management decisions.

\section{Conclusions}

In this study cohort, patients who had undergone MVD had a longer duration of pain control than those who had undergone MVD+Rhiz or SRS. While fewer complications were observed in the SRS cohort, the complication rates seen for the MVD and MVD+Rhiz cohorts were consistent with published rates for younger patients. Our results suggest that both MVD and SRS are effective options for the elderly and that treatment choice should be tailored to each patient's unique condition and treatment goals. We hope our experience can help counsel physicians and patients on available treatment options.

\section{References}

1. Klun B. Microvascular decompression and partial sensory rhizotomy in the treatment of trigeminal neuralgia: personal experience with 220 patients. Neurosurgery. 1992;30(1): 49-52.

2. Raygor KP, Lee A, Chang EF. Treatment of idiopathic trigeminal neuralgia in the elderly. In: Sagher O, Levin EL, Pilitsis JG, eds. Pain Neurosurgery. Oxford University Press; 2019:9-12.

3. Barker FG II, Jannetta PJ, Bissonette DJ, et al. The long-term outcome of microvascular decompression for trigeminal neuralgia. N Engl J Med. 1996;334(17):1077-1083.

4. Ferroli P, Acerbi F, Tomei M, et al. Advanced age as a contra- 
indication to microvascular decompression for drug-resistant trigeminal neuralgia: evidence of prejudice? Neurol Sci. 2010;31(1):23-28.

5. Günther T, Gerganov VM, Stieglitz L, et al. Microvascular decompression for trigeminal neuralgia in the elderly: longterm treatment outcome and comparison with younger patients. Neurosurgery. 2009;65(3):477-482.

6. Sekula RF Jr, Frederickson AM, Jannetta PJ, et al. Microvascular decompression for elderly patients with trigeminal neuralgia: a prospective study and systematic review with meta-analysis. J Neurosurg. 2011;114(1):172-179.

7. Sekula RF, Marchan EM, Fletcher LH, et al. Microvascular decompression for trigeminal neuralgia in elderly patients. $J$ Neurosurg. 2008;108(4):689-691.

8. Cohen J, Mousavi SH, Faraji AH, et al. Stereotactic radiosurgery as initial surgical management for elderly patients with trigeminal neuralgia. Stereotact Funct Neurosurg. 2017;95(3): $158-165$.

9. Mousavi SH, Niranjan A, Huang MJ, et al. Early radiosurgery provides superior pain relief for trigeminal neuralgia patients. Neurology. 2015;85(24):2159-2165.

10. Wang DD, Raygor KP, Cage TA, et al. Prospective comparison of long-term pain relief rates after first-time microvascular decompression and stereotactic radiosurgery for trigeminal neuralgia. J Neurosurg. 2018;128(1):68-77.

11. Oh IH, Choi SK, Park BJ, et al. The treatment outcome of elderly patients with idiopathic trigeminal neuralgia: microvascular decompression versus Gamma Knife radiosurgery. $J$ Korean Neurosurg Soc. 2008;44(4):199-204.

12. Yu R, Wang C, Qu C, et al. Study on the Therapeutic effects of trigeminal neuralgia with microvascular decompression and stereotactic Gamma Knife surgery in the elderly. J Craniofac Surg. 2019;30(1):e77-e80.

13. Eller JL, Raslan AM, Burchiel KJ. Trigeminal neuralgia: definition and classification. Neurosurg Focus. 2005;18(5):E3.

14. Burchiel KJ, Slavin KV. On the natural history of trigeminal neuralgia. Neurosurgery. 2000;46(1):152-155.

15. Burchiel KJ. A new classification for facial pain. Neurosurgery. 2003;53(5):1164-1167.

16. Tomlinson SB, Piper K, Kimmell KT, Vates GE. Preoperative frailty score for 30-day morbidity and mortality after cranial neurosurgery. World Neurosurg. 2017;107:959-965.

17. Rogers CL, Shetter AG, Fiedler JA, et al. Gamma Knife radiosurgery for trigeminal neuralgia: the initial experience of the Barrow Neurological Institute. Int J Radiat Oncol Biol Phys. 2000;47(4):1013-1019.

18. Raygor KP, Wang DD, Ward MM, et al. Long-term pain outcomes for recurrent idiopathic trigeminal neuralgia after stereotactic radiosurgery: a prospective comparison of firsttime microvascular decompression and repeat stereotactic radiosurgery. J Neurosurg. 2019;131(4):1207-1215.

19. Bederson JB, Wilson CB. Evaluation of microvascular decompression and partial sensory rhizotomy in 252 cases of trigeminal neuralgia. J Neurosurg. 1989;71(3):359-367.

20. Jannetta PJ, McLaughlin MR, Casey KF. Technique of microvascular decompression. Technical note. Neurosurg Focus. 2005;18(5):E5.

21. McLaughlin MR, Jannetta PJ, Clyde BL, et al. Microvascular decompression of cranial nerves: lessons learned after 4400 operations. J Neurosurg. 1999;90(1):1-8.

22. Youngerman BE, Neugut AI, Yang J, et al. The modified frailty index and 30-day adverse events in oncologic neurosurgery. J Neurooncol. 2018;136(1):197-206.

23. Martínez-Anda JJ, Barges-Coll J, Ponce-Gomez JA, et al. Surgical management of trigeminal neuralgia in elderly pa- tients using a small retrosigmoidal approach: analysis of efficacy and safety. J Neurol Surg A Cent Eur Neurosurg. 2015; 76(1):39-45.

24. Mastronardi L, Caputi F, Rinaldi A, et al. Typical trigeminal neuralgia: comparison of results between patients older and younger than 65 years operated on with microvascular decompression by retrosigmoid approach. J Neurol Surg A Cent Eur Neurosurg. 2020;81(1):28-32.

25. Phan K, Rao PJ, Dexter M. Microvascular decompression for elderly patients with trigeminal neuralgia. J Clin Neurosci. 2016;29:7-14.

26. Rughani AI, Dumont TM, Lin CT, et al. Safety of microvascular decompression for trigeminal neuralgia in the elderly. Clinical article. J Neurosurg. 2011:115(2):202-209.

27. Yang DB, Wang ZM, Jiang DY, Chen HC. The efficacy and safety of microvascular decompression for idiopathic trigeminal neuralgia in patients older than 65 years. J Craniofac Surg. 2014;25(4):1393-1396.

28. Ko AL, Ozpinar A, Lee A, et al. Long-term efficacy and safety of internal neurolysis for trigeminal neuralgia without neurovascular compression. J Neurosurg. 2015;122(5):10481057.

29. Karam SD, Tai A, Wooster M, et al. Trigeminal neuralgia treatment outcomes following Gamma Knife radiosurgery with a minimum 3-year follow-up. J Radiat Oncol. 2014;3: 125-130.

30. Marshall K, Chan MD, McCoy TP, et al. Predictive variables for the successful treatment of trigeminal neuralgia with Gamma Knife radiosurgery. Neurosurgery. 2012;70(3): $566-573$

31. Karam SD, Tai A, Snider JW, et al. Refractory trigeminal neuralgia treatment outcomes following CyberKnife radiosurgery. Radiat Oncol. 2014;9:257.

32. Tang Y-Z, Jin D, Bian J-J, et al. Long-term outcome of computed tomography-guided percutaneous radiofrequency thermocoagulation for classic trigeminal neuralgia patients older than 70 years. J Craniofac Surg. 2014;25(4):1292-1295.

33. Ying X, Wang H, Deng S, et al. Long-term outcome of percutaneous balloon compression for trigeminal neuralgia patients elder than 80 years: a STROBE-compliant article. Medicine (Baltimore). 2017;96(39):e8199.

34. Lunsford LD. Treatment of tic douloureux by percutaneous retrogasserian glycerol injection. JAMA. 1982;248(4): 449-453.

\section{Disclosures}

The authors report no conflict of interest concerning the materials or methods used in this study or the findings specified in this paper.

\section{Author Contributions}

Conception and design: Raygor. Acquisition of data: all authors. Analysis and interpretation of data: Raygor. Drafting the article: Raygor. Critically revising the article: Chang, Raygor, Barbaro. Reviewed submitted version of manuscript: Chang, Raygor, Barbaro. Statistical analysis: Raygor. Administrative/technical/material support: Ward. Study supervision: Chang, Barbaro.

\section{Correspondence}

Edward F. Chang: University of California, San Francisco, CA. changed@neurosurg.ucsf.edu. 